\title{
Experimental Study on Bubble Acoustic Characteristics of Elliptical Nozzles
}

\author{
Shanhao Cong ${ }^{1}$ and Jingting Liu ${ }^{1,2, *}$ \\ ${ }^{1}$ School of Mechanical Engineering, Shandong University, 17923, Jingshi Road, Jinan, Shandong, \\ China \\ ${ }^{2}$ Key Laboratory of High Efficiency and Clean Mechanical Manufacture, Ministry of Education, \\ Shandong University, 17923, Jingshi Road, Jinan, Shandong, China
}

\begin{abstract}
Bubble acoustic signals were reported experimentally using five different nozzles with the same exit cross-sections. The bubble morphology was captured by a home-made experiment setup, which could synchronously obtain images of two perpendicular directions. Short-Time Fourier Transform and synchronization of video and audio were adopted to analyse the acoustic signal. Results demonstrated that the sound pressure of symmetrical bubble formation was higher than that of asymmetrical bubble formation, while the sound pressure of asymmetrical bubble formation was higher than that of the bubble with an unapparent necking fracture detached from the nozzle of the aspect ratio is $4: 1$. Furthermore, the last bubble of bubble chains would change the shape and decay rate of the acoustic signal due to the difference in the period of bubble formation. These studies can provide more effective information to predict the leaks from undersea gas pipelines, seabed methane reserves, and carbon capture and storage facilities.
\end{abstract}

\section{Introduction}

The phenomenon of bubble flow widely exists in many fields of subjects and industries, such as nature, national defence, energy development, and environmental protection. For example, aerosols caused by the breakup of bubbles play an important role in the transportation and exchange of materials in the ocean-atmosphere ecosystem [1]. In addition, the release of greenhouse gases from shallow seabed gas and deep-sea cold springs has a profound impact on global warming [2]. In recent years, submarine oil and gas pipeline leakage accidents occur frequently, causing serious pollution and huge economic losses. If a timely prediction can be made in the early stages of an accident, the damage can be minimized. Therefore, it is of significance for energy development, accident prevention, and environmental protection to study the bubble flow mechanism and the internal relationship between bubble flow field and sound field.

\footnotetext{
* Corresponding author: liujingting@sdu.edu.cn
} 
Bubble acoustic characteristics are an important means of bubble kinematics characterization. In recent years, acoustic emission technology has played an increasingly important role in experimental research, industry, and life due to its advantages of noncontact and high precision. Rayleigh [3] is almost the first researcher to study sounds emitted by bubbles. Minnaert [4] came up with the natural frequency equation of a single, millimetre-sized, linearly oscillating bubble,

$$
f_{0}=\frac{1}{R_{0}}\left(\frac{3 \gamma P_{A}}{4 \pi^{2} \rho}\right)^{1 / 2}
$$

Minnaert's equation is considered to be a simplified solution of the Rayleigh equation, which builds a bridge between bubble morphology and acoustic signal. Bubbles produce an acoustic signal owing to the oscillation of the gas in the bubble. Subsequently, Strasberg [5], Lamb [6], and Longuet-Higgins [7] attempted to establish the internal relationship between ideal bubbles and the simultaneous radiation of distorted bubble morphological changes, making it possible for acoustic signals to characterize bubble morphological changes. Longuet-Higgins [8] analysed the process of raindrops falling to the water surface and summarized the causes of bubble radial oscillation excitation. The relationship between bubble oscillation and some parameters (pressure, shape, velocity field) is revealed. With deeply understand of the simple bubble acoustic principle, more and more researchers concentrate on the investigation of the collective oscillations of a bubble cloud. Yoon [9] demonstrated that the bubble plumes generated by breaking waves can be a strong source of relatively low frequency $(<1 \mathrm{kHz})$ ambient noise in a column of bubble experiment. Zorana [10] used the concepts and techniques of condensed matter physics to explore the effects of viscous damping, the distance between bubbles, geometric disorder, size of the bubbles, and size of the cloud. The complexity of bubble clouds makes it difficult to do more deep work, so some researchers turn to the simple situation in bubble chain or fewer bubbles. Shima [11] deduced an equation for the natural frequency of the oscillation of two spherical bubbles in an incompressible liquid. It was found that the natural frequency between bubbles increased with the increase of the ratio of bubble radius to space. Manasseh [12] used hydrophones to measure the sound pressure around the bubble chain and analysed the change of pulse waveform along the direction of the bubble chain. The result suggested that the sound field had a strong anisotropy.

It is known that an elliptical nozzle with a larger aspect ratio is similar to a crack on the pipeline. At present, the researches on elliptical nozzles mainly focus on the aspects of heat transfer, reducing noise, and abrasive jet machining. To improve the combustion efficiency of diesel engines, many researchers used elliptical nozzles to enhance spray characteristics and mixing quality. Yu [13] proved that the elliptical spray exhibited larger spray areas than the circular spray, which suggested that using an elliptical orifice was helpful to increase the mixture quality of fuel and air. Molina [14] has done some work about the use of elliptical orifices on the inner nozzle flow and cavitation development in diesel injector nozzles. However, the majority of the studies involving elliptical nozzles have been limited to gaseous jets. Arghode [15] investigated to study the characteristics of exit gas jet and their effect on the sound pressure levels over a range of jet momentum conditions. Hao [16] found that sound pressure levels for elliptical nozzle were much lower than those of other nozzles over the frequency bands of $0.6-4 \mathrm{kHz}$. They both tried to determine different characteristics of the jet by acoustic signal or sound pressure.

Though studies on elliptical nozzles and acoustic signals are becoming deeper, there is less to explore the relationship between bubble morphology and acoustic signal at a low speed. Research on elliptical nozzles mainly focuses on the characteristics of heat transfer. 
Using elliptical nozzles to study the influence of boundary on bubble detachment provides a new direction to grasp the rules in regular and simple conditions. It can offer a reference for the future research on pipeline cracks. In this paper, five different kinds of elliptical nozzles with different aspect ratios were used to simulate the fracture shape of submarine pipelines. Owing to spatial constraints, the special bubble morphology and acoustic characteristics were analysed emphatically when the aspect ratio is $4: 1$.

\section{Methodology}

\subsection{Experimental setup}

The layout of experimental devices is shown in Figure 1. Air is generated by a syringe pump, which is transferred through pipe and nozzle into the acrylic tank. The size of the acrylic tank is $0.3 \times 0.3 \times 0.5 \mathrm{~m}$ and the length is designed more than 20 times of bubble diameter, which can reduce acoustic reverberation [17]. The liquid in the tank is water at a room temperature of $20^{\circ} \mathrm{C}$. The height of the water level is $150 \mathrm{~mm}$. Two lamps with diffuser screens provide adequate and homogenous illumination. A special image system is used to make the high-speed camera $\left(1295 \mathrm{fps}, 1280 \times 720 \mathrm{pixel} / \mathrm{cm}^{2}\right)$ synchronously obtain the image in two perpendicular directions. The imaging system consists of two groups of mirrors, called fixed mirrors and adjustable mirrors. Reflected twice by two mirrors, the light changes its angle by 45 degrees. Two hydrophones (Reson TC4013, -209.9dB) are placed $5 \mathrm{~cm}$ from the nozzle to obtain an acoustic signal that will be converted into a voltage signal in data acquisition. The high-speed camera and data acquisition are connected to the trigger that can synchronously record the data of image and acoustic signals in the computer. Five nozzles with different aspect ratios (AR) are used in the experiment, which keeps the same outlet section. The shape of the five types of nozzles is shown in Figure 2.

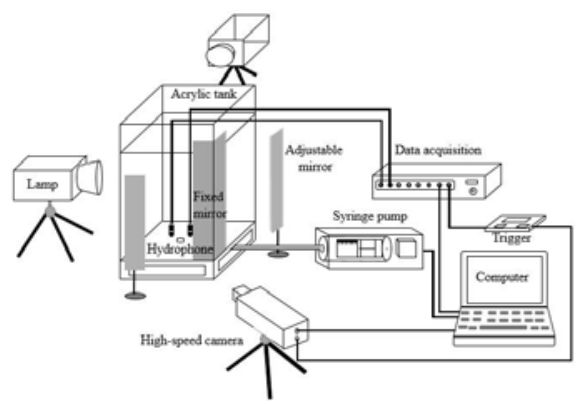

Fig. 1. The layout of experimental devices.

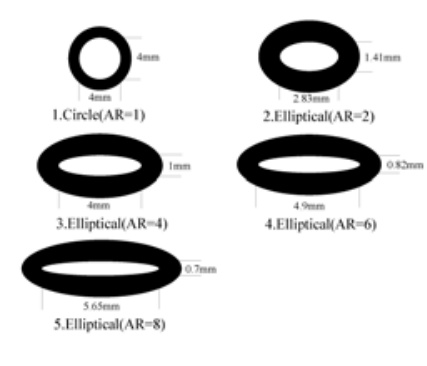

Fig. 2. The shape of the five types of nozzles.

\subsection{Experimental methods}

Two experimental methods are applied to explore the rules between bubble morphology and acoustic signal. One is the synchronization of video and audio, the other one is ShortTime Fourier Transform (STFT). Synchronization of video and audio is to realize the correspondence of image and acoustic signals that are obtained from the high-speed camera and hydrophones. For instance, the synchronization of video and audio is shown in Figure 3. The range of band-pass filter is $300-3000 \mathrm{~Hz}$. The necking fracture leads to a pulse of 
acoustic pressure in the flow field when the bubble leaves the nozzle. The theory makes the synchronization possible and precise.

STFT is one of the most effective methods to obtain the center frequency of the acoustic signal at any specific moment through the MATLAB program. The equation is as follows,

$$
X(k)=\sum_{n=0}^{n-1} x(n) e^{-\frac{j 2 \pi n k}{N}}, k=0,1, \cdots, N-1
$$

Time-frequency analysis technics based on STFT are applied in the experiment. It is shown in Figure 4. Hamming window (window length 512, window function overlap rate $50 \%$ ) is used to segment the experimental audio signal and identify the central frequency band of the sound signal.

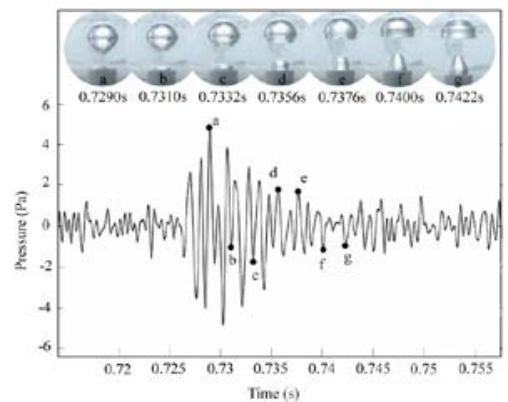

Fig. 3. Synchronization of video and audio.

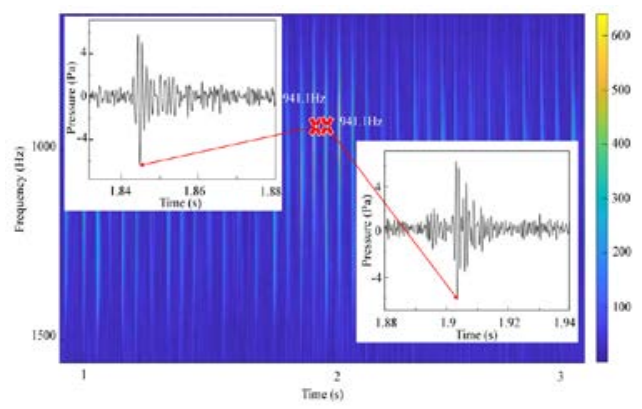

Fig. 4. Spectrogram.

\section{Results and discussion}

\subsection{Bubble morphology generated from five different nozzles}

Different sizes of nozzles can lead to different bubble morphology and acoustic signal during the process of bubble formation. As the aspect ratio increases, the phenomenon has become more complex. When the aspect ratio is beyond four, the bubbles were generated like a chain, not just a single bubble at all. The images of bubbles generated from different nozzles are shown in Figure 5. In the last 100 years, researchers have studied regular rules of acoustic bubbles that are generated in a circular nozzle, but they still need to explore the different irregular nozzle. Although the acoustic signal is more complex, there are still many rules existing behind the phenomenon. 


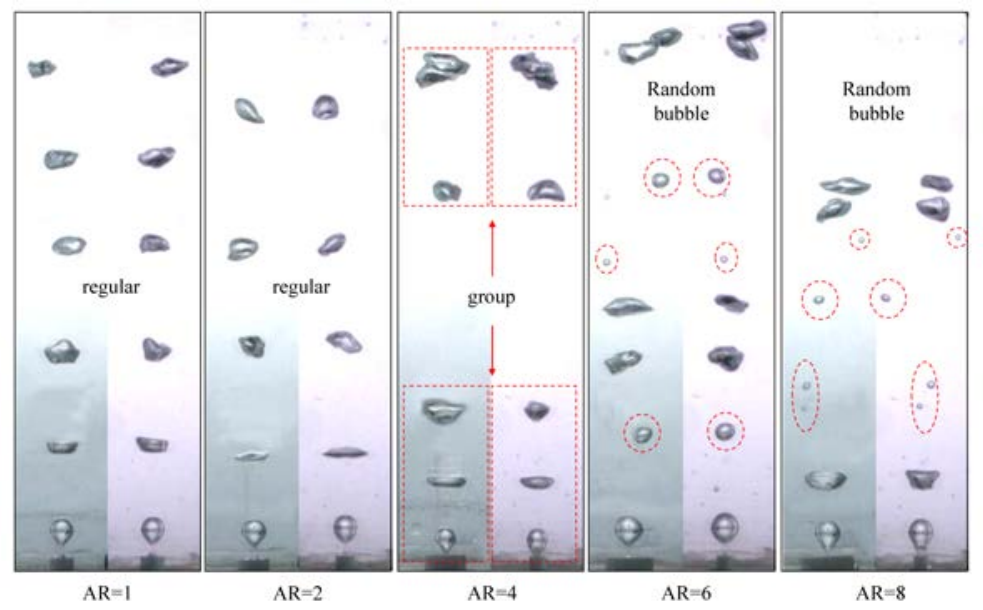

Fig. 5. The progress of bubble formation and rising in five different nozzles.

\subsection{Special acoustic signal related to bubble morphology}

When the aspect ratio is $4: 1$, there is an interesting phenomenon. A group of bubble chains has different acoustic signals that correspond to different bubble morphology. In common conditions, a group of bubble chains consists of three bubbles as shown in Figure 6. The first two bubbles are bigger than the third. The first bubble is similar to the second bubble, but the acoustic signals are exactly different from each other. There are some differences between the first two bubbles. As it is shown in Figure 6, the pressure of the second bubble is much larger than the first one. The reason that causes the difference arises more attention. STFT is used to obtain the frequency of bubble formation. The frequency of point a and c are both $867.2 \mathrm{~Hz}$, which represent the moment that two bubbles leave the nozzle respectively. STFT spectrogram of bubble formation is shown in Figure 7. According to Minnaert's equation, bubble diameter is inversely proportional to frequency. If the frequency is the same, the diameter should be the same too. The theory confirms bubble image and acoustic signal are correct. The difference may be caused by other reasons. Through careful comparison and observation of the first two bubbles, we found that the second bubble was more symmetrical than the first. The upper right part of the first bubble was clearly protruded outward. The asymmetry was becoming more apparent during the bubble rising. In contrast, the second bubble was more symmetrical in both two directions. The acoustic signal was generated by the bubble oscillation, the jet that formed at the moment of bubble leaving nozzle was the main cause of bubble oscillation. The jet that was generated by bubble formation was affected by the nozzle and flow flied. 


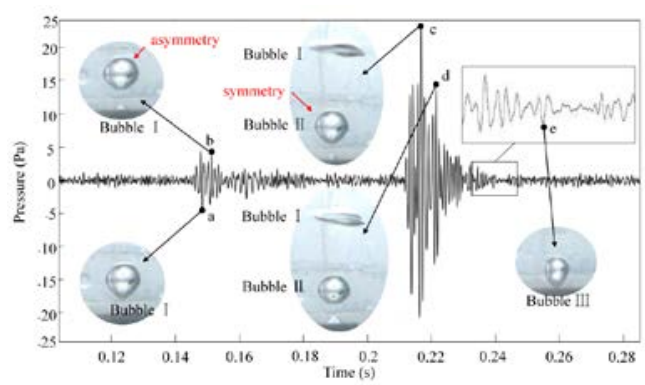

Fig. 6. The acoustic signal of bubble formation and rising under the aspect ratio is $4: 1$.

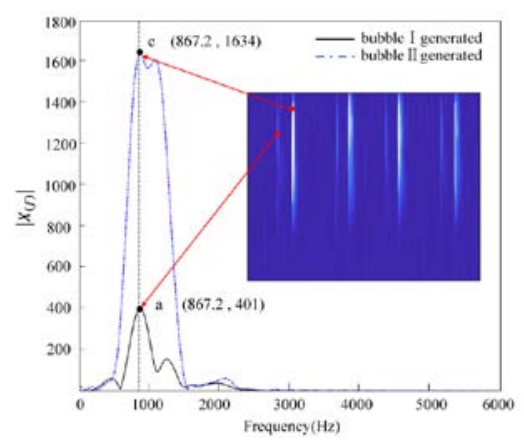

Fig. 7. STFT spectrogram of bubble formation.

\subsection{Effect of acoustic signal between bubbles}

The bubble morphology at the moment of bubble leaving the nozzle is closely connected to the level of sound pressure. The third bubble has an unapparent necking fracture, so it can't generate high-level sound pressure. A special group including just two bubbles was compared. The acoustic signal is shown in Figure 8. The acoustic signal of the first bubble is like a decaying sine wave. The acoustic signal caused by the second bubble is too small to change the waveform of the first.

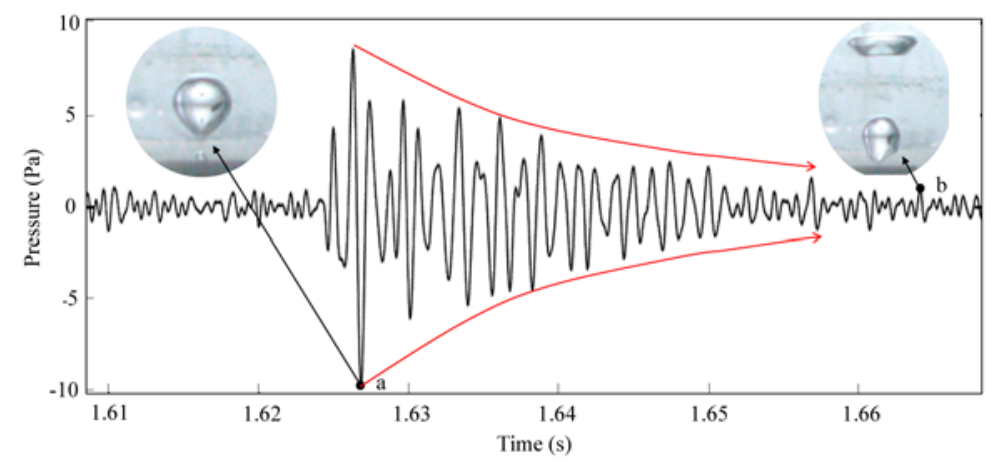

Fig. 8. The acoustic signal caused by a group of two bubbles. 

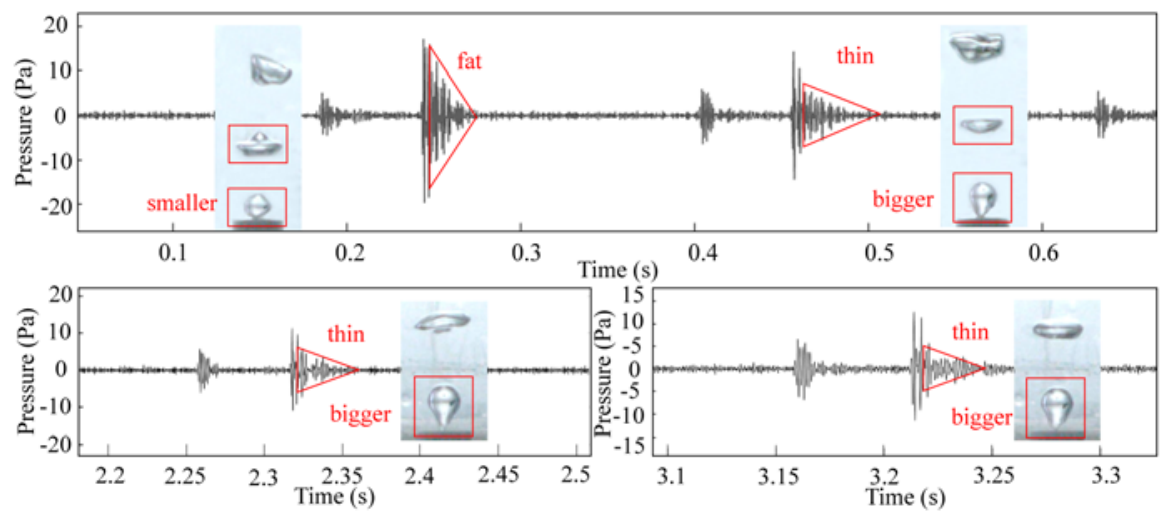

Fig. 9. Sound pressure change and image contrast diagram.

In a group of three bubbles, though the third bubble can't generate high-level sound pressure, it still makes a great impact on the acoustic signal. In Figure 9, the way and morphology of the third bubble leaving nozzle affect the waveform of the acoustic signal. When the third bubble with an unapparent necking fracture is smaller at the moment of leaving the nozzle, the acoustic signal is just like a "fat" triangle. When the third bubble is bigger, the acoustic signal is just like a "thin" triangle. If the third bubble is smaller, it will be generated following the second bubble as fast as possible. The decreased speed of sound pressure is faster. But when the third bubble is larger, the decrease speed of sound pressure is slower. The morphology of the second bubble is observed at the moment that the third bubble was generated. The degree of second bubble development is different. The second bubble with a smaller third bubble is developing faster than the second bubble with a larger third bubble.

\section{Conclusion}

This paper studied the effects of elliptical nozzles on bubble morphology and acoustic signal. It started with an experiment of bubble formation progress from five different elliptical nozzles, paying more attention to the result of the nozzle with the aspect ratio of $4: 1$. It was shown that the bubble morphology and acoustic were becoming more complex with the increase of aspect ratio. Under the nozzle of the aspect ratio 4:1, the sound pressure of symmetrical bubble formation is higher than the sound pressure of asymmetrical bubble formation. The sound pressure of asymmetrical bubble formation is higher than the sound pressure of bubble with an unapparent necking fracture. Though the bubble with an unapparent necking fracture has a lower sound pressure, the third bubble morphology can also influence the acoustic signal in a group of three bubbles. When the third bubble is small, the acoustic signal is just like a "fat" triangle. When the third bubble is big, the acoustic signal is just like a "thin" triangle. The acoustic signal of the second bubble with a smaller third bubble is developing faster than that of the second bubble with a larger third bubble. Irregular bubble morphology and the way of bubble formation are closer to the environmental condition. The shape of the nozzle can affect the bubble noise indirectly. It will become more efficient to predict and analyse the special acoustic signal at the cracks of submarine pipelines. 


\section{Acknowledgments}

This work was supported by the National Natural Science Foundation of China (No. 52006126); Shandong Provincial Natural Science Foundation (No. ZR2020QE193).

\section{References}

[1] Quinn P and Asher W 2006 Bulletin of the American Meteorological Society vol $87 \mathrm{p}$ 505-507.

[2] Xijie Y, Huaiyang Z, Qunhui Y 2015 Acta Oceanologica Sinic vol 87 p 69-75.

[3] Rayleigh L 1917 Philosophical Magazine vol 200 p 504-507.

[4] Minnaert M 1933 The London, Edinburgh, and Dublin Philosophical Magazine and Journal of Science vol 16 p 235-248.

[5] Strasberg M 1956 The Journal of the Acoustical Society of America vol 28 p 20-26.

[6] Lamb H 1932 Hydrodynamics 6th edn Cambridge University Press.

[7] Longuet-Higgins M S 1989 Journal of Fluid Mechanics vol 201 p 525-541.

[8] Longuet-Higgins M S 1990 Journal of Fluid Mechanics vol 214 p 395-410.

[9] Yoon S W 1991 The Journal of the Acoustical Society of America vol 89 p 700-706.

[10] Zeravcic Z, Lohse D, Van Saarloos W 2011 Journal of Fluid Mechanics vol 680 p 114149.

[11] Shima A 1971 Journal of Basic Engineering vol 93 p 426-431.

[12] Manasseh R, Nikolovska A, Ooi A 2004 Journal of Sound and Vibration vol $278 \mathrm{p}$ 807-823.

[13] Yu S, Yin B, Deng W 2019 Fuel vol 236 p 1474-1482.

[14] Molina S, Salvador F J, Carreres M 2014 Energy Conversion and Management vol 79 p 114-127.

[15] Arghode V K and Gupta A K 2012 Applied Energy vol 89 p 246-253.

[16] Hao Z and Zhou Z 2012 Applied Mechanics and Materials vol 204-208 p 4620-4623.

[17] Manasseh R, Lafontaine R F, Davy J 2000 Experiments in fluids vol 30 p 672-682. 